\title{
Micromagnetics Simulation of Asymmetric Pseudo-Spin Valve Dots
}

\author{
N. Dao, ${ }^{1}$ C. A. Ross, ${ }^{2}$ F. J. Castaño, ${ }^{2}$ M. J. Donahue, ${ }^{3}$ and S. L. Whittenburg ${ }^{1}$ \\ ${ }^{1}$ Department of Chemistry/AMRI, University of New Orleans, New Orleans, LA 70148, U.S.A. \\ ${ }^{2}$ Department of Materials Science and Engineering, Massachusetts Institute of Technology, \\ Cambridge, MA 02139, U.S.A. \\ ${ }^{3}$ National Institute of Standards and Technology, Gaithersburg, MD 20899, U.S.A.
}

\begin{abstract}
We present our recent simulation results for $\mathrm{Ni}_{79} \mathrm{Fe}_{21}(5 \mathrm{~nm}) / \mathrm{Cu}(3 \mathrm{~nm}) / \mathrm{Co}(4 \mathrm{~nm})$ pseudo-spin valves. These simulations have been conducted on several different aspect ratios of rectangular dots. Distinct switches of the two magnetic layers were observed. At smaller aspect ratios, magnetization reversal proceeds through a leaf state in the soft layer and a flower state in the hard layer. For larger aspect ratios, reversal proceeds by nucleation and annihilation of a domain wall. Our simulations show a reasonable agreement with the experimental results. Differences between the experimental and simulation results are discussed.
\end{abstract}

\section{INTRODUCTION}

Research in Magnetoresistive Random Access Memory (MRAM) has been very active since MRAM provides a competitive memory compared to Dynamic Random Access Memory (DRAM) and Flash technology [1]. The promising structures for future MRAM are magnetic tunnel junctions and pseudo-spin valves [2-4]. There have been recent reports of fabrication of pseudo-spin valves with sub-100 nm dimensions [5-7]. By using micromagnetics simulation, the magnetic states occurring during the switching process can be determined, which will aid in designing a magnetic device. In this study, we apply micromagnetics to examine the magnetization reversal process in rectangular pseudo-spin valve elements. The soft layer is permalloy and the hard layer is cobalt. The switching mechanism of these magnetic layers will be addressed.

\section{PROCEDURE}

Our calculations were performed on $\mathrm{Ni}_{79} \mathrm{Fe}_{21}$ ( $5 \mathrm{~nm}$, bottom layer)/Cu (3 nm)/Co (4 nm, top layer) pseudo-spin valves. The experimental pseudo-spin valve of $\mathrm{Ni}_{79} \mathrm{Fe}_{21}(6 \mathrm{~nm}) / \mathrm{Cu}(3 \mathrm{~nm}) /$ Co $(4 \mathrm{~nm}) / \mathrm{Cu}(4 \mathrm{~nm})$ was deposited by DC sputtering and patterned by interference lithography into rectangular dot arrays $[5,7]$ with widths of $70-90 \mathrm{~nm}$ and aspect ratios of 1.5-10. The experimental thicknesses of 6,3 , and $4 \mathrm{~nm}$ are nominal and the "real" thickness is probably different. Slightly better agreement with the experimental loops was obtained by using a $5 \mathrm{~nm}$ permalloy layer. The spacing between the dots in an array is three times the width and two times the length of the dot. The magnetostatic interaction between these dots is negligible. In this work, we simulated only a single dot. The simulated hysteresis loops were obtained from dots with a range of aspect ratios from 1.8 to 7.9. The experimental loops were obtained using 
MicroMag ${ }^{\mathrm{TM}} 2900$ alternating gradient magnetometer.

We used 3D OOMMF (Object-Oriented MicroMagnetic Framework) [8] to obtain the simulated hysteresis loops. These calculations are based on using the Landau-Lifshitz (LL) equation, where the effective field includes the anisotropy, applied, exchange, and selfmagnetostatic fields. The exchange coupling through RKKY coupling between the soft and hard layers can also be included in the calculation of the local field.

The anisotropy field is a first order approximation with anisotropy constants of $\mathrm{K}_{1}=5.2 \times 10^{5}$ $\mathrm{J} / \mathrm{m}^{3}$ for cobalt and $\mathrm{K}_{1}=-600 \mathrm{~J} / \mathrm{m}^{3}$ for permalloy. The anisotropy constant for permalloy is in agreement with the experimentally reported value [9]. The easy axes were taken to be aligned perpendicular to the magnetic element plane. The exchange field was computed using the six nearest neighbors, and the default exchange coupling constants of $A=3.0 \times 10^{-11} \mathrm{~J} / \mathrm{m}$ for cobalt and $\mathrm{A}=1.3 \times 10^{-11} \mathrm{~J} / \mathrm{m}$ for permalloy were used. The default values of the saturation magnetization, $\mathrm{M}_{\mathrm{s}}=1.4 \times 10^{6} \mathrm{~A} / \mathrm{m}$ for cobalt and $\mathrm{M}_{\mathrm{s}}=8.6 \times 10^{5} \mathrm{~A} / \mathrm{m}$ for permalloy were also used. OOMMF uses Newell's method to calculate the magnetostatic interaction, where the magnetization is constant inside the discretized cell [10]. The cell size was $5 \mathrm{~nm} \times 5 \mathrm{~nm} \times 1 \mathrm{~nm}$ in $\mathrm{x}, \mathrm{y}$, and $\mathrm{z}$-directions. The major hysteresis loop was obtained by following the equilibrium states of the magnetization obtained from solution of the LL equation by the forward Euler method. The applied field was along the long axis of the rectangular dots. All simulations began with a random configuration of spins and the saturated state was obtained by slowing increasing the applied field to the starting field of the reported hysteresis loops.

\section{RESULTS AND DISCUSSION}

For the range of aspect ratios used in this study, there are two switching modes. One involves transitions through a flower state, and the other through a formation of domain wall in the middle of the dot. We will show two hysteresis loops, each of which represents one of these types. The full details of the switching process will be discussed for the dots with small aspect ratios. For the dots with large aspect ratios, only the reversals at the two switches will be provided. The magnetic domains are the same before and after reversal for all aspect ratios.

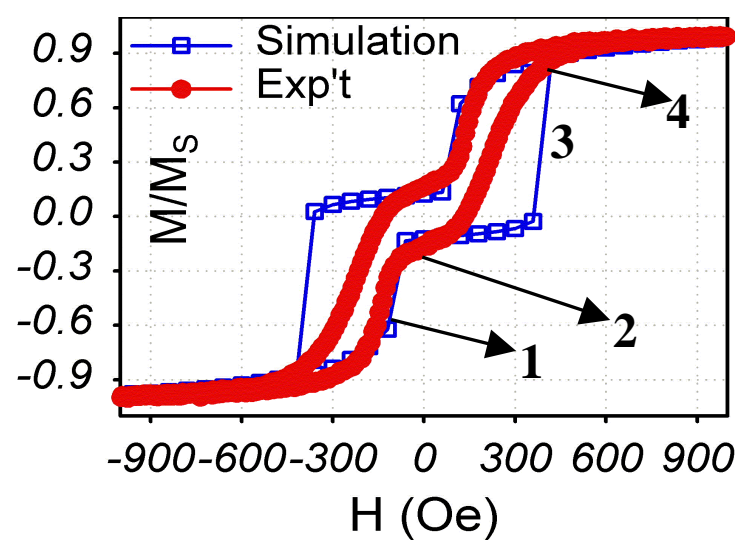

Figure 1. The simulation loop (hollow square) for the rectangular $90 \mathrm{~nm} \times 160 \mathrm{~nm}$ dot and the experimental loop (solid circle) for the $90 \mathrm{~nm}$ x $170 \mathrm{~nm}$ dot array. The numerical notations correspond to the magnetic states in figure 2 . 
At small aspect ratios such as a dot of $90 \mathrm{~nm} \times 160 \mathrm{~nm}$ (figure 1), the switch of the soft layer occurs before the loop comes to the remanent state. The switching field is in good agreement with that obtained from the experimental hysteresis loop. The magnetization reversal inside the dot starts with the formation of an S state in both layers. As the soft layer begins to switch, its magnetization forms a leaf (L) state as shown in figure 2.1 and indicated in the simulation loop of figure 1 at the state 1. This state is similar to leaf states noted previously in soft magnetic square elements [11]. The top layer at this point is in a tilted S state. The spins in the soft layer rotate nearly coherently and switch before the hysteresis loop reaches its remanent state.
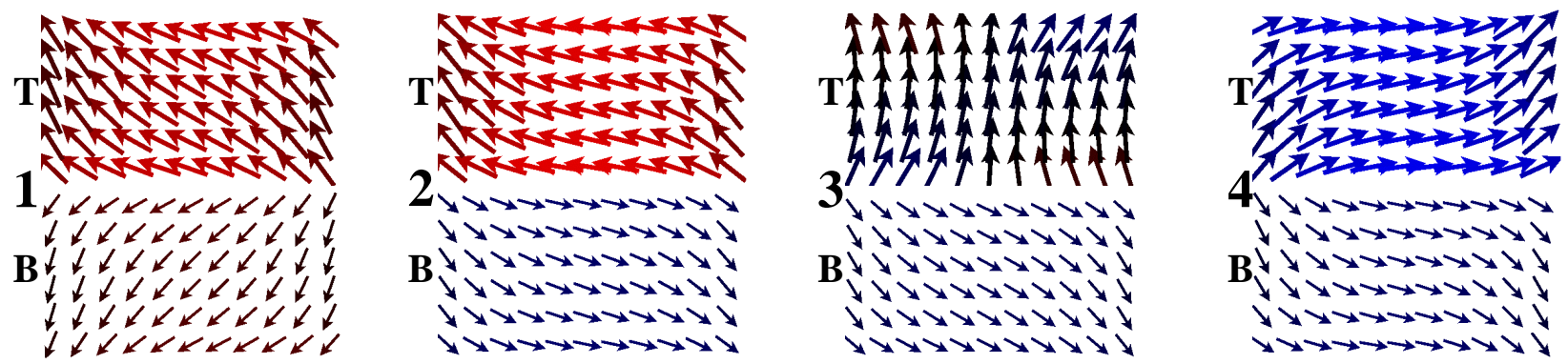

Figure 2. The magnetic domains correspond to notations in figure 1. Every third spin is shown. The numbers inserted in the front of two magnetic domains for one state are as the same numbers indicated in figure 1. B stands for the bottom layer (soft layer), and T stands for the top layer (hard layer).

At remanence, the magnetization spins between the bottom and top layers are anti-parallel (figure 2.2) in an S state. The net magnetization at this point is not at zero since the magnetization of the hard layer is higher than the soft layer. Through $\mathbf{1}$ and $\mathbf{2}$ (figure 2), we can see that spins in the soft layer move counterclockwise while in the hard layer spins rotate clockwise into 3. This opposite movement between two layers is due to the opposite sense of the $\mathrm{S}$ state in the two layers in the initial spin configuration. At $\mathbf{3}$ (figure 2), the magnetic state of the hard layer displays a flower $(\mathrm{F})$ state and the tilted $\mathrm{S}$ in the soft layer. This corresponds to the second switch. After this switch, magnetic domains for both layers are in the $\mathrm{S}$ with spins at the edges having an opposite sense (figure 2.4).

Experimentally, the coercivity of the hard layer increases with aspect ratio while in the soft layer coercivity changes between positive and negative field values. The simulated switching field of the hard layer is always found to be higher than the experimentally obtained switching field for all aspect ratios. We have examined simulation artifacts, such aligning the applied field slightly off-axis and have included long-range RKKY coupling of the two magnetic layers. None of these corrections shifts the simulated switching field by enough to explain the discrepancy with the experimental value. It appears that some process is leading to an early switch in the experimental loops. It is interesting to note that in all of the simulation loops the second switching field occurs near the field where the magnetization reaches its saturation value. We will discuss this discrepancy later in the article.

The dot of $90 \mathrm{~nm} \times 450 \mathrm{~nm}$ represents the second type of switching behavior (figure 3 ). In figure 3 the view is from the plus z-direction looking down on each layer. The switching between two layers is well defined in both of the experimental and simulation loops. The simulated switching field of the soft layer is larger than the experimental switching field. The 
switching field of the soft layer in this high aspect ratio sample occurs after remanence. At remanence, the majority of simulation spins are still at the saturated state in an elongated S state in both layers. For the reversal of both the soft and hard layers, the magnetization reverses through a domain wall (DW) as shown in the insets 1 and 2. The inset 1 also shows that the hard layer has a leaf state in the first switch, indicating that the applied field is not strong enough to reverse the hard layer. After the soft layer has switched, the hard layer begins to form a domain wall, leading to reversal (the inset 2).

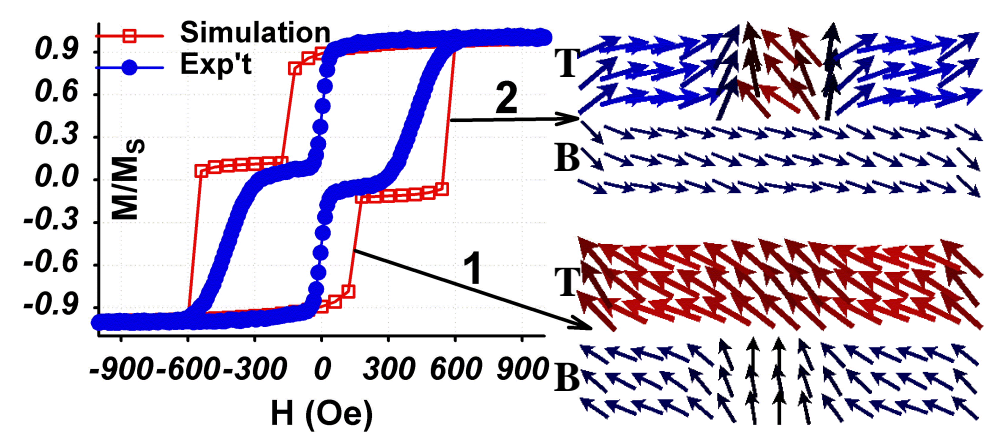

Figure 3. The simulation hysteresis loop (hollow rectangle) for the rectangular $90 \mathrm{~nm} \times 450 \mathrm{~nm}$ dot and the experimental loop (solid circle) for the rectangular $90 \mathrm{~nm}$ x $450 \mathrm{~nm}$ dot array. Every $6^{\text {th }}$ spin is shown. At $\mathbf{1}$ and $\mathbf{2}$, non-equilibrium magnetization patterns during two switching events are inset. B stands for the bottom (soft) layer. T stands for the top (hard) layer.

The simulated switching field of the soft layer is different from the experimental switching field as shown in figure 3 for the aspect ratio of 5. However, when the simulation dot size was $90 \mathrm{~nm} \times 350 \mathrm{~nm}$, instead of $90 \mathrm{~nm}$ x $450 \mathrm{~nm}$, this switching field is significantly closer to the experimental switching field of the soft layer. For all aspect ratios (table I), the switching field of the soft layer is consistently larger when the aspect ratio is increased in the simulation. The (*) in table I indicates aspect ratios with available experimental data. The dimensions of each dot size are given in $\mathrm{nm}$. An L indicates a leaf state, an $\mathrm{F}$ for flower state, and a DW for domain wall in the dot. As showed in table I, when the aspect ratio is large the reversal of magnetization occurs through the formation of a domain wall. The transition from a single domain state (L/F) to a domain wall (DW/DW) occurs somewhere between an aspect ratio of 2.63 and 3.89 .

Table I. Magnetic domains in the soft and hard layers at different aspect ratios (AR).

\begin{tabular}{|c|c|c|c|c|c|c|c|}
\hline $\begin{array}{c}\text { AR } \\
\text { Layex }\end{array}$ & $\begin{array}{c}1.78^{*} \\
(90 \times 160)\end{array}$ & $\begin{array}{c}2.22 \\
(90 \times 200)\end{array}$ & $\begin{array}{c}2.57^{*} \\
(70 \times 180)\end{array}$ & $\begin{array}{c}2.63^{*} \\
(80 \times 210)\end{array}$ & $\begin{array}{c}3.89 \\
(90 \times 350)\end{array}$ & $\begin{array}{c}5.00^{*} \\
(90 \times 450)\end{array}$ & $\begin{array}{c}7.86^{*} \\
(70 \times 550)\end{array}$ \\
\hline Soft & $\mathbf{L}$ & $\mathbf{L}$ & $\mathbf{L}$ & $\mathbf{F}$ & $\mathbf{D W}$ & $\mathbf{D W}$ & $\mathbf{D W}$ \\
\hline Hard & $\mathbf{F}$ & $\mathbf{F}$ & $\mathbf{F}$ & $\mathbf{F}$ & $\mathbf{D W}$ & $\mathbf{D W}$ & $\mathbf{D W}$ \\
\hline
\end{tabular}

In the experimental loops, for all aspect ratios except the arrays of the $70 \mathrm{~nm}$ short axis dots, 
the first switch leads to a state whose magnetization is the same sign as the saturated state for that part of the loop. This is easily seen in figure 1. For the half-loop from +900 Oe to -900 Oe, the initial state has $\mathrm{M} / \mathrm{M}_{\mathrm{s}}$ close to 1.0 and the $\mathrm{M} / \mathrm{M}_{\mathrm{s}}$ value after the first switch is greater than zero. For the arrays of the $70 \mathrm{~nm}$ short axis dots, the experimental hysteresis loops have the $\mathrm{M} / \mathrm{M}_{\mathrm{S}}$ value below zero for this half-loop after the first switch. The simulation does not reproduce this.

For rectangular dots, magnetization domains can form one of the two states, $\mathrm{C}$ or $\mathrm{S}$, sometimes referred to as the end domains [12]. A C state will favor the magnetostatic energy while an S state will favor the exchange energy. This helps explain the discrepancy of the samples with the $70 \mathrm{~nm}$-short axes. When the $\mathrm{C}$ state forms in the dot, the hysteresis loop may not display a distinct switching of the two layers since the layers can trap a domain wall, even for small dot sizes [13]. We calculated the hysteresis loops with the applied field $5^{\circ}-10^{\circ}$ off from the long axis. The hysteresis loops became closely matched with the experimental loops. The reasons for the effect have been discussed [14].

Our simulation loops showed a higher $\mathrm{dM} / \mathrm{dH}$ than the experimental loops, especially at the switching field of the hard layer. This difference arises from the variability in shape and size of the experimental dots in an array. Small defects in the experimental dots can lead to lower switching fields. Our calculations also don't take into account the slight slope of the edge of the experimental dots. This slope produces a hard layer that is slightly smaller than the soft layer. Also, the thickness of these layers is nominal; therefore, the real thickness is probably different from the thickness used in the simulation. These two factors would yield differences in the $M / M_{s}$ value after the first switch in the simulation loops relative to the experimental loops. Actually, the pre-patterned film [15] gave a similar $\mathrm{dM} / \mathrm{dH}$ as in the simulation loop. Finally, the experimental films are polycrystalline with no strong crystallographic texture. All of these factors combine to explain discrepancies between the simulation and experimental results. However, it should be noted that there is good general agreement with the loop shapes and, more importantly, the trends in the coercivities with aspect ratio.

One other possible term that could be included in the local field in the simulations is the longrange exchange or RKKY coupling between the two layers. A bilinear coupling constant of $8 \times 10^{-5} \mathrm{~J} / \mathrm{m}^{2}$ was used to test on the $90 \mathrm{~nm} \times 160 \mathrm{~nm}$ dot. This constant is smaller than the experimental approximation for both bilinear and biquadratic coupling in a similar system with different layer thickness [16]. Including this long-range coupling made the switching field of the soft layer occur prior to remanence much earlier, and the switching field of the hard layer moved to higher field. This suggests that the interlayer exchange coupling is extremely small for the $\mathrm{Ni}_{79} \mathrm{Fe}_{21} / \mathrm{Cu}(3 \mathrm{~nm}) / \mathrm{Co}$ system. RKKY coupling was not included in the results presented here.

\section{CONCLUSIONS}

Overall, our simulations are in reasonable agreement with the experimental results. The general shape of the hysteresis loops compare well with the experimental loops and the trend of the coercivities of both the hard and soft layer switching with aspect ratio is reproduced by the simulation. The switching of the bottom and top layers is observed. When the dot is small and when the dot starts in an S state, the soft layer switches before remanence. The magnetization reversal occurs via rotation of a leaf configuration in the soft layer and a flower configuration in the hard layer for samples up to an aspect ratio of 2.63. This switching mechanism shows that 
these samples have a promise for high- density magneto-electronic devices. For higher aspect ratios, the switching of the layers occurs through the formation and annihilation of domain wall. The switching fields of the soft layer increase consistently as the aspect ratio increases. The end domains can be forced to an $\mathrm{S}$ state by taking the applied field off from the long axis of the dot a few degrees. There are no vortices in the samples at the range of these dot sizes, making them potential candidates for MRAM device [4]. Magnetic elements that reverse via rotation are better candidates for MRAM devices because the device switches at a reproducible switching field. Elements that reverse via magnetic domains containing vortices or domain wells usually display variability in their switching fields leading to non-reproducible magnetization reversal characteristics.

\section{ACKNOWLEDGMENTS}

The authors acknowledge the support of this work by AMRI through DoD/DARPA Grant No. MDA972-97-0003, NSF, and the Louisiana Board of Regents via NSF/LEQSF (2001-04)-R1103.

\section{REFERENCES}

1. S. A. Wolf and D. Treger, IEEE Trans. Magn. 36, 2748 (2000).

2. S. Tehrani, B. Engel, J. M. Slaughter, E. Chen, M. DeHerrera, M. Durlam, P. Naji, R. Whig, J. Janesky, and J. Calder, IEEE Trans. Magn. 36, 2752 (2000).

3. S. Gidor, B. -U. Runge, A. C. Marley, and S. S. P. Parkin, Science 281, 797 (1998).

4. J. M. Daughton. A. V. Pohm, R. T. Fayfield, and C. H. Smith, J. Phys. D 32, R169 (1999).

5. F. J. Castaño, Y. Hao, M. Hwang, C. A. Ross, B. Vogeli, H. I. Smith, and S. Haratani, Appl. Phys. Lett. 79, 1504 (2001).

6. F. J. Albert, J. A. Katine, R. A. Buhrman, and D. C. Ralph, Appl. Phys. Lett. 77, 3809 (2000).

7. F. J. Castaño, Y. Hao. C. A. Ross, B. Vogeli, H. I. Smith, and S. Haratani, J. Appl. Phys. (in press).

8. M. J. Donahue and D. G. Porter, Interagency Report NISTIR 6376, National Institute of Standards and Technology, Gaithersburg, MD (Sept 1999).

9. R. A. McCurrie, Ferromagnetic Materials: Structure and Properties, Academic Press, San Diego 1994, p. 29.

10. A. J. Newell, W. Williams, and D. J. Dunlop, J. Geophys. Res. 98, 9551 (1993).

11. R. P. Cowburn and M. E. Welland, Phys. Rev. B 58, 9217 (1998).

12. J. Gadbois, J. -G. Zhu, W. Vavra, and A. Hurst, IEEE Trans. Magn. 34, 1066 (1998).

13. N. Dao, Y. Hao, C. A. Ross, M. L. Malkinski, J. -Q. Wang, and S. L. Whittenburg, J. Appl. Phys. (in press).

14. R. D. McMichael, M. J. Donahue, D. G. Porter, and J. Eicke, J. Appl. Phys. 89, 7603 (2001); A. Hubert and W. Rave, Phys. Stat. Sol. B 211, 815 (1999).

15. F. J. Castaño, Y. Hao, S. Haratani, C. A. Ross, B. Vogeli, M. Walsh, and H. I. Smith, IEEE Trans. Magn. 37, 2073 (2001).

16. T. Tanuma, S. Takahashi, A. Maeda, M. Kume, and K. Kuroki, IEEE Trans. Magn. 31, 3955 (1995). 